\title{
Near-field flow structure of a confined wall jet on flat and concave rough walls
}

\author{
I. ALBAYRAK ${ }^{1}$, E. J. HOPFINGER ${ }^{2} \uparrow$ AND U. LEMMIN \\ ${ }^{1}$ Laboratory of Environmental Hydraulics (LHE), Ecole Polytechnique Fédérale de Lausanne (EPFL), \\ Lausanne, Switzerland \\ ismail.albayrak@epfl.ch; ulrich.lemmin@epfl.ch \\ ${ }^{2}$ LEGI-CNRS/UJF, Grenoble Cedex, France \\ emil.hopfinger@hmg.inpg.fr
}

(Received 10 September 2007 and in revised form 26 February 2008)

Experimental results are presented of the mean flow and turbulence characteristics in the near field of a plane wall jet issuing from a nozzle onto flat and concave walls consisting of fixed sand beds. This is a flow configuration of interest for sediment erosion, also referred to as scouring. The measurements were made with an acoustic profiler that gives access to the three components of the instantaneous velocities. For the flat-wall flow, it is shown that the outer-layer spatial growth rate and the maxima of the Reynolds stresses approach the values accepted for the far field of a wall jet at a downstream distance $x / b_{0} \approx 8$. These maxima are only about half the values of a plane free jet. This reduction in Reynolds stresses is also observed in the shear-layer region, $x / b_{0}<6$, where the Reynolds shear stress is about half the value of a free shear layer. At distances $x / b_{0}>11$, the maximum Reynolds shear stress approaches the value of a plane free jet. This change in Reynolds stresses is related to the mean vertical velocity that is negative for $x / b_{0}<8$ and positive further downstream. The evolution of the inner region of the wall jet is found to be in good agreement with a previous model that explicitly includes the roughness length.

On the concave wall, the mean flow and the Reynolds stresses are drastically changed by the adverse pressure gradient and especially by the development of Görtler vortices. On the downslope side of the scour hole, the flow is nearly separating with the wall shear stress tending to zero, whereas on the upslope side, the wall-friction coefficient is increased by a factor of about two by Görtler vortices. These vortices extend well into the outer layer and, just above the wall, cause a substantial increase in Reynolds shear stress.

\section{Introduction}

Wall jets are encountered in aeronautical, industrial and hydraulic applications. Typical examples in hydraulic engineering are jets issuing from sluice gates and from dam bottom outlets, and jets used in the dredging of sand in rivers. In these situations of sediment erosion, it is the near field of the wall jet that is of importance (Rajaratnam 1981; Hogg, Huppert \& Dade 1997; Hopfinger et al. 2004). Aeronautical and industrial applications, such as film cooling, are mainly concerned with the far field. Because of the widespread interest in film cooling, most studies of wall jets have

\footnotetext{
$\dagger$ Author to whom correspondence should be addressed.
} 
focused on the far-field flow structure (Launder \& Rodi 1983; Wygnanski, Katz \& Horev 1992; George et al. 2000). The experiments of Rajaratnam (1981) cover the near and far field of a wall jet on a rough wall, but only mean flow quantities have been measured.

The turbulent wall jet is also of fundamental interest. It is composed of two interacting flow regions, namely the inner wall-bounded flow and the outer free-shear flow. Wall friction is important in the inner layer which is similar to a boundary layer, whereas the outer layer behaves like a plane jet. Abrahamsson, Johansson \& Löfdahl (1994) defined the point of maximum velocity of the wall jet as the limit between these two flow zones. However, as has been pointed out in most papers on wall jets (Launder \& Rodi 1981), the interaction between the large turbulent scales in the outer layer and the smaller scales in the wall layer creates a complex flow field that displaces the position of zero shear stress from the position of maximum velocity (zero mean velocity gradient) toward the wall. This implies that the energy-containing eddies are not in equilibrium with the mean flow near the location of the velocity maximum. Launder \& Rodi (1981) presented a detailed review of the topic. Wygnasnski et al. (1992) investigated the similarity of the far field of a wall jet and George et al. (2000) developed a general similarity theory.

Erosion prevention aimed at the protection of hydraulic structures in relation to jet effects is discussed by Mason \& Arumugam (1985). Rajaratnam (1981) and Chatterjee, Ghosh \& Chatterjee (1994) showed that a jet issuing onto mobile sediment beds causes significant erosion immediately downstream of the jet exit. These authors and others have proposed correlations for the depth of erosion, the scour hole depth, as a function of the jet characteristics and the bed material. Hogg et al. (1997) developed a theory of erosion by a wall jet that depends critically on the near-field turbulence characteristics of a turbulent wall jet on a rough wall. Hogg et al. used a mass conservation equation and assumed that the critical Shields parameter for flat beds could be extended to concave beds. Hopfinger et al. (2004, hereinafter referred to as HKGL) examined in detail the Hogg et al. model and showed that, in fact, the wall shear stress is likely to be increased by Görtler vortices that develop when the sediment bed is sufficiently concave and the Görtler number is large. The near stop in erosion is then a result of upslope transport in the scour hole by the enhanced erosion potential of the flow owing to Görtler vortices and downslope avalanching.

The question is to what extent do Görtler vortices contribute to the wall shear stress. Experiments with heat transfer on concave surfaces indicate that the Nusselt number can increase by 100-150\% (Mayle, Blair \& Kopper 1979; Floryan 1991). By analogy between momentum and heat transfer, an increase in heat flux at the wall implies also an increase in wall shear stress. Barlow \& Johnston (1985) reported an increase in wall shear stress on a concave wall of $40 \%$ above the flat-wall value. In general, longitudinal vortices in a turbulent wall flow affect the wall shear stress. Kravchenko, Choi \& Moin (1993) demonstrated the close correlation between wall shear stress and near-wall streamwise vortices in turbulent boundary layers and Nino \& Garcia (1996) showed that these near-wall vortices on a loose sediment bed can cause the sediment to accumulate in the low-speed-streak regions near the wall. Larger-scale longitudinal vortices in developed turbulent open-channel flow that are related to corner vortices have a similar effect, but at the scale of the flow depth. However, these vortices are relatively weak; the secondary flow velocity is about $2 \%$ of the maximum mean velocity (Tamburrino \& Gulliver 1999; Albayrak \& Lemmin 2007) and the increase in wall shear stress is at most $10 \%$ (Albayrak 2007). As expected, the wall shear stress is larger in the downwash regions and smaller in the upwash zones 
between the vortices. The strength of Görtler vortices can be an order of magnitude greater than the streamwise vortices in a straight channel. The simple model used by HKGL suggests an increase in Reynolds shear stress close to the wall by about a factor of four to five with respect to a flat wall. Measurements by Kobayashi $\&$ Fujisawa (1983), made in the wall region of a turbulent wall jet on a concave smooth wall support this model. Because of the rarity of such results, and of the uncertainty about the contribution of these vortices to the shear stress, it is of interest to conduct measurements of mean flow and Reynolds stresses in the configuration used by HKGL. We have conducted such measurements, first on a flat fixed sand bed and then on a concave fixed sand bed of roughness equal to the mobile sand bed of HKGL and shape corresponding to the quasi-steady scour-hole shape. Since the flow is developing, the results obtained on the flat wall are crucial for the interpretation of the results obtained on the concave wall.

Measurements were made with a three-dimensional acoustic Doppler velocity profiler (ADVP) for flow conditions identical to those of HKGL. This profiler is nonintrusive, no particles have to be introduced and it has the advantage that the three components of the instantaneous velocity can be measured rapidly over the whole flow depth. The ADVP consists of two independent working subsystems in the longitudinal and spanwise flow sections, which make a redundant measurement of the instantaneous vertical velocity field in order to correct the data. Hurther \& Lemmin (2000) demonstrated that the AVDP mean flow and turbulence data compare well with data obtained by more conventional techniques.

The experimental set-up and procedures are described in $\S 2$. The mean flow field and turbulence characteristics on the horizontal wall are presented in $\S 3$, including an analysis of the inner-layer flow development in terms of the scaling proposed by Hogg et al. (1997). The results on the concave wall are discussed in $\S 4$, emphasizing the changes with respect to the flat wall. In the Appendix the results on the flat wall are analysed in terms of the flow over a backward-facing step.

\section{Experimental conditions}

\subsection{Installation}

The experiments were conducted in a glass-sided horizontal flume $17 \mathrm{~m}$ long, 0.5 $\mathrm{m}$ wide and $0.8 \mathrm{~m}$ deep. Figure 1 shows the experimental set-up and the definition of the flow parameters. The upstream and the downstream ends of the flume were raised in order to create a cavity $0.35 \mathrm{~m}$ deep and $3.8 \mathrm{~m}$ long that was filled with uniformly graded sand of mean diameter $d_{50}=2 \mathrm{~mm}$ that is equal to the roughness length $k_{e}$ used in the theoretical analysis. For the flat-wall experiments (figure $1 a$ ) the surface particles were fixed in place by spraying them with glue in order to create a fixed-bed section of roughness equivalent to a mobile sand bed. The concave wall shape (figure $1 b$ ) corresponds to the shape that a mobile bed takes owing to scouring when the depth has reached a quasi-steady state as defined by HKGL. After this depth was reached, the flow was stopped and, after the water had been drained, the surface sand particles were fixed in place by spraying them with glue.

The jet issued from a nozzle of $b_{0}=5 \mathrm{~cm}$ underneath a vertical sluice gate located at $L_{f}=10 \mathrm{~cm}$ upstream of the beginning of the fixed sand beds. A constant water discharge, $Q$, was maintained by controlling $\Delta h=h_{1}-h_{2}$. The upstream water depth $h_{1}$ was kept constant during the experiments by an overflow gate, and the downstream water depth $h_{2}$ was controlled by adjusting a gate at the downstream end of the flume. The channel has a zero slope angle. 
(a)
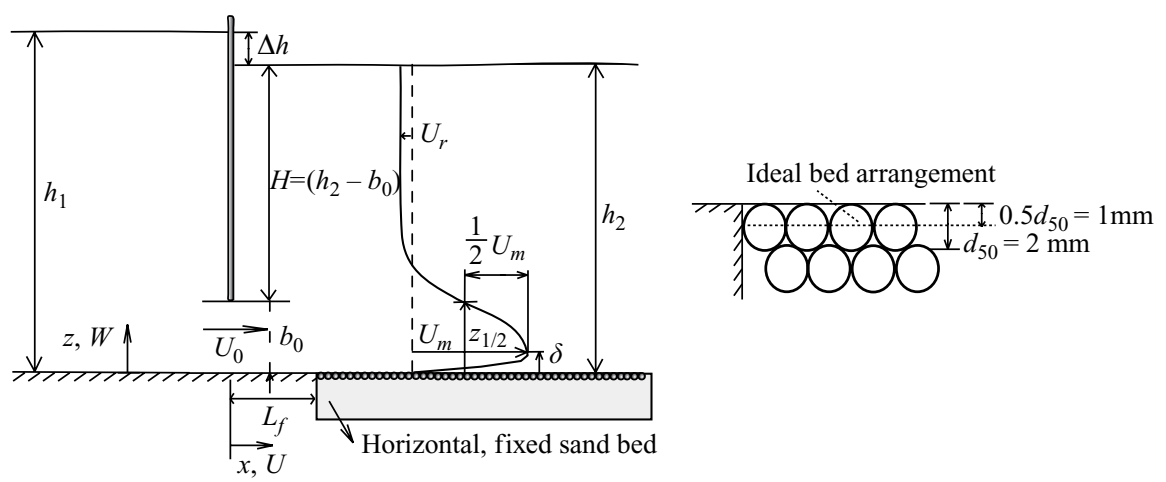

(b)

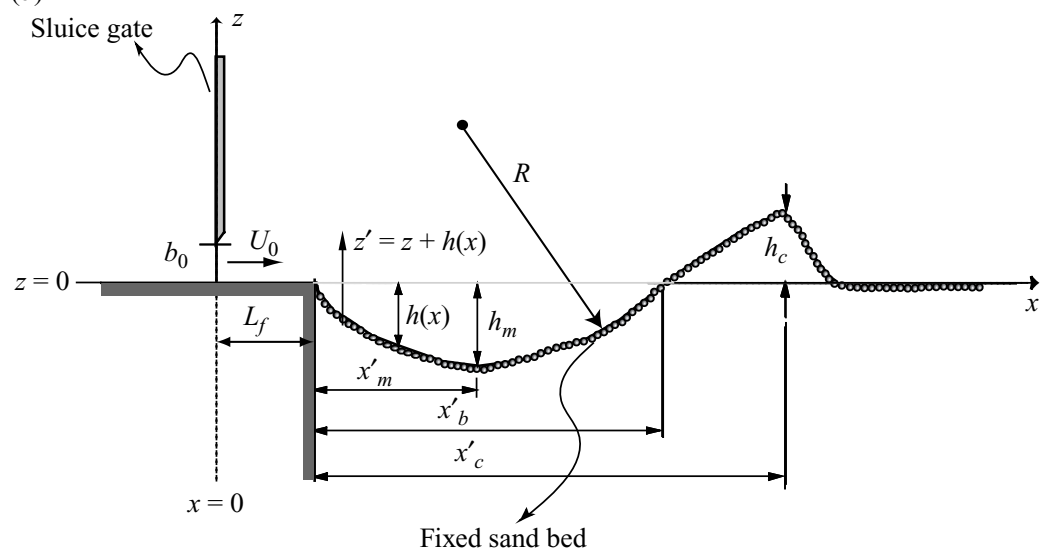

FIGURE 1. Schematic of the plane wall jet and of the downstream fixed beds. $(a)$ Flat wall with definitions of flow variables, and $(b)$ concave wall shape of depth $h(x)$ and maximum depth, $h_{m}=6.4 \mathrm{~cm}$, at downstream position $x_{m}^{\prime}=15 \mathrm{~cm} ; x_{b}^{\prime}=30 \mathrm{~cm}$ is the position of the zero $z$ level crossing; $h_{c}=-7.3 \mathrm{~cm}$ is the downstream hill height at $x_{c}^{\prime}=43 \mathrm{~cm} . R=20.5 \mathrm{~cm}$ is the radius of the curvature of the concave wall starting at $x=L_{f}=10 \mathrm{~cm}$ downstream of sluice gate; $x^{\prime}=x-L_{f}$. The origin of $z^{\prime}$ is at the scour hole boundary with $z^{\prime}=z+h(x)$.

Experiments were conducted with a difference in upstream and downstream water levels, respectively $h_{1}=24.7 \mathrm{~cm}$ and $h_{2}=22.1 \mathrm{~cm}$, of $\Delta h=h_{1}-h_{2}=2.6 \mathrm{~cm}$, giving a nominal nozzle inlet velocity $U_{0}=\sqrt{2 g \Delta h}=0.71 \mathrm{~m} \mathrm{~s}^{-1}$. The Reynolds number is $R e=U_{0} b_{0} / v=3.53 \times 10^{4}$ and the Froude number is $F r=U_{0} / \sqrt{g b_{0}}=1.014$. Table 1 gives a summary of the flow conditions. The origins of $z$ on the flat wall and $z^{\prime}$ on the concave wall are taken at the top of the sand grains. The experimental conditions of the flow over the concave wall are identical to those defined in HKGL. The sand roughness is $k_{e}=d_{50}=2 \mathrm{~mm}$.

\subsection{Velocity measurements}

The mean and turbulent velocities were measured with the three-dimensional ADVP, developed at the Environmental Hydraulics Laboratory (LHE) at the EPFL. This instrument measures the quasi-instantaneous velocity vector at a large number of points within the water column. Its space and time resolution are, respectively, $3 \mathrm{~mm}$ and about $0.032 \mathrm{~s}$. This is sufficient for determining the turbulent quantities with good accuracy relatively close to the wall. A detailed description of the ADVP 


$\begin{array}{lccccccccc} & h_{1} & h_{2} & \Delta h & R & G_{T} \text { Görtler } & T & U_{0} & & \\ \text { Test } & (\mathrm{cm}) & (\mathrm{cm}) & (\mathrm{cm}) & (\mathrm{cm}) & \text { number } & (\mathrm{s}) & \left(\mathrm{cm} \mathrm{s}^{-1}\right) & \operatorname{Re}=\frac{U_{0} b_{0}}{v} & F r=\frac{U_{0}}{\sqrt{g b_{0}}} \\ & & & & & & & & & \\ \text { Flat wall } & 24.7 & 22.1 & 2.6 & - & - & 180 & 71 & 3.35 \times 10^{4} & 1.014 \\ \text { Concave wall } & 24.7 & 22.1 & 2.6 & 20.5 & 2.86 & 180 & 71 & 3.35 \times 10^{4} & 1.014\end{array}$

TABLE 1. Test conditions for flat and concave boundaries. The Görtler number is defined by $G_{T}=31 \sqrt{\theta / R}$ where $\theta$ is the momentum thickness of the boundary layer (HKGL). $T$ is the measuring time.

system is given by Lhermitte \& Lemmin (1994) and Rolland \& Lemmin (1997). The acoustic profiler makes use of the backscattering echo of an ultrasonic pulse produced by moving micro contaminants present in the water. The Doppler frequency shift observed between the emitted and the received signal is proportional to the fluid particle velocity and its direction. For the present measurements, a multistatic configuration of the ADVP was used, consisting of four receivers that surround the emitter, providing one redundancy in the three-dimensional velocity component calculation. This redundancy eliminates the signal noise and improves the data quality (Hurther \& Lemmin 2000). Pulse repetition frequencies (PRF) of $1667 \mathrm{~Hz}$ and 1111 $\mathrm{Hz}$, with a number of pulse pairs (NPP) equal to 32 were used, which results in velocity data acquisition frequencies of 52 and $35 \mathrm{~Hz}$, respectively. At each measured profile, data were collected for about $3 \mathrm{~min}$. Even though a low degree of aliasing was expected, a dealiasing algorithm (Franca \& Lemmin 2006) was applied. Measurements were made for the whole longitudinal range and the whole depth, with a spatial resolution of $3 \mathrm{~mm}$. All measurements were made at the centre of the channel. Owing to the interference of the ADVP housing with the sluice gate, the measurements were started at a distance of $19.75 \mathrm{~cm}$ from the gate.

On a mobile sediment bed with erosion, turbulence measurements with the ADVP in the wall region are not reliable because the moving sand grains are seen by the ADVP as obstacles and create fixed echoes in the signal. This results in bad quality turbulence data.

\subsection{Wall shear stress measurements}

Wall shear stress measurements were conducted with a sensor developed at the LHE (Shen, Song \& Lemmin 1996). It is based on the hot-film principle and has constant sensitivity in variable-temperature flow. The hot-film element is mounted flush with the top of the fixed sand grains. The voltage required to maintain a constant temperature of the thermal element is related to the wall shear stress $\tau_{b}=\left.\mu(\partial U / \partial z)\right|_{z=0}$ in the form,

$$
\tau_{b}^{1 / 2}=A E^{2}+B
$$

where $\tau_{b}$ is the wall shear stress, $A$ and $B$ are constants which have to be found by calibrating the hot-film probe, and $E$ is the voltage required to maintain constant temperature. Using (1), the mean wall shear stress was measured at several streamwise positions. The error in the wall shear stress measured with the hot-film probe is larger on a rough wall than on a smooth wall. From calibrations in a straight channel, the error in shear stress on the rough wall was evaluated to be $\pm 5 \%$. 


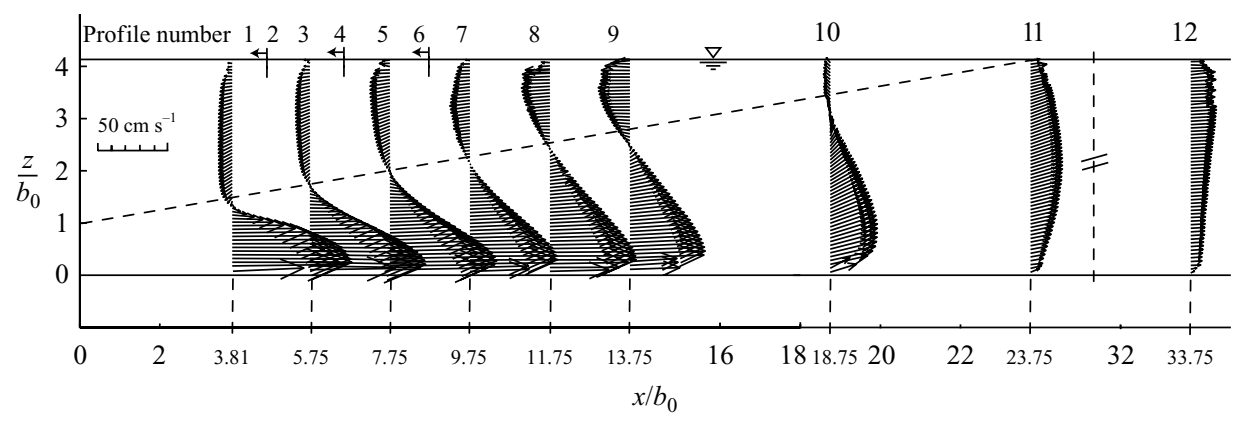

FIGURE 2. Mean velocity vectors in the $(x, z)$-plane. The position $x=0$ is at the sluice gate and the sand roughness starts at $x / b_{0}=2$. For clarity, profiles 2,4 and 6 have been omitted. The dashed line delineates backflow. The velocity scale on the left shows $\sqrt{U^{2}+W^{2}}$.

\section{Flow characteristics on the flat wall}

\subsection{Evolution of mean flow quantities}

Figure 2 shows the mean velocity vectors in the $(x, z)$-plane at the centre of the channel at nine downstream positions, starting at $x / b_{0}=3.81$ and ending at $x / b_{0}=33.75$. These mean velocity vectors have been obtained from the instantaneous, threedimensional ADVP signals.

The streamwise maximum velocity $U_{m}$ is larger than $U_{0}$, when $x / b_{0}<8$, indicating that just downstream of the sluice gate a favourable pressure gradient exists that is related to the return flow. This is clearly seen in figure 3 where $U_{m} / U_{0}$ is plotted as a function of $x / b_{0}$ together with the spatial growth of the outer layer, characterized by the position $z_{1 / 2}$, which is defined to be the position at which $U=U_{m} / 2$. For $x / b_{0}<5$, the ratio between maximum streamwise velocity and nominal nozzle or sluice gate velocity is $U_{m} / U_{0} \cong 1.14$. The velocity maximum $U_{m}$ starts to decay when $x / b_{0}>6$. Momentum conservation gives a variation $U_{m} / U_{0} \propto\left(x / b_{0}\right)^{-1 / 2}$; this variation is indicated in figure 3 where the prefactor of 2.7 is close to the prefactor 2.8 determined by Hogg et al. (1997) using Rajaratnam's (1967) data. As will be shown below, because of bottom friction, the exponent $m<0.5$ in $U_{m} \sim x^{-m}$.

The spatial growth rate of the outer layer tends to merge into the value $\mathrm{d} z_{1 / 2} / \mathrm{d} x=$ 0.073 that is generally accepted for the growth rate of the far-field outer layer of a wall jet without external flow (Launder \& Rodi 1983). Figure 3 indicates that up to distance $x / b_{0} \approx 8$ the growth rate of the outer layer is much less. This distance corresponds roughly to the flow-development region referred to as the potential core length. By definition, the potential core corresponds to the distance required for the penetration of the mixing layer from above and the turbulent boundary layer from below over the initial jet width $b_{0}$. When taking a mixing-layer growth of $\delta / x \approx 0.17$ and a rough-wall boundary-layer growth $\delta_{b} \approx 0.4 x\left(U_{0} x / \nu\right)^{-1 / 5}$, we obtain the expression $(0.085 x+0.03 x) / b_{0} \approx 1$, giving a value $x / b_{0} \approx 8$ for the potential core length. This estimated length can be reduced if the thickness of the turbulent boundary layer is increased by adding an initial thickness at the nozzle. By the same reasoning, the potential core length of a plane free jet issuing from a nozzle of width $b_{0}$ is given by the distance where the mixing layers (of growth $\delta / x \approx 0.17$ ) merge. This gives $x / b_{0} \approx 6$, as is observed in the experiments.

Concerning the position of the velocity maximum with respect to the wall, the present data are best fitted by $\delta / b_{0} \cong 0.024 x / b_{0}+0.13$. The virtual origin is, therefore, at $x_{0} \cong-5.4 b_{0}$ so that $\delta / b_{0} \cong 0.024\left(x-x_{0}\right) / b_{0}$. Note that when no adjustment is 


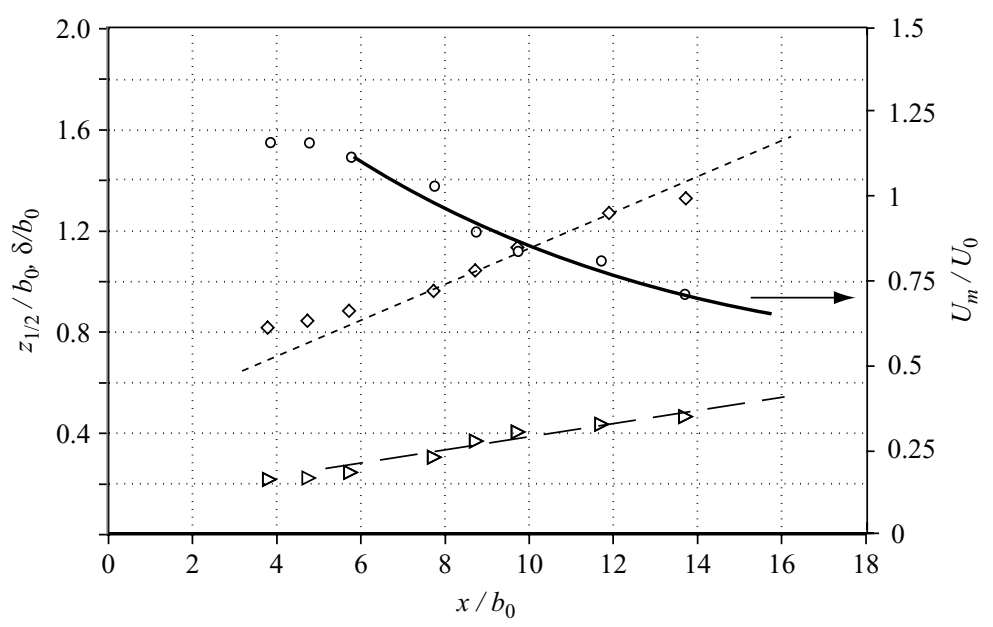

FIGURE 3. Variation of dimensionless mean velocity, $U_{m} / U_{0}$, and spatial growths rates of the outer layer, $z_{1 / 2} / b_{0}$, and inner layer, $\delta / b_{0}$, with downstream distance $x / b_{0} . \bigcirc, U_{m} / U_{0}$; $\diamond, z_{1 / 2} / b_{0} ; \triangleright, \delta / b_{0} ;-, U_{m} / U_{0}=2.7\left(x / b_{0}\right)^{-1 / 2} ;---, z_{1 / 2} / b_{0}=0.073 x+0.39 ;-—-$ $\delta / b_{0} \cong 0.024 x / b_{0}+0.13$.

made for the virtual origin, the growth of $\delta$ has to be fitted by a power law that accounts for the initial flow-development region (Wygnanski et al. 1992; Hogg et al. 1997). In the linear fit with a virtual origin, the value of 0.024 is smaller by a factor of two than the prefactor suggested by Hogg et al. (but no virtual origin was included in their fit), and is larger by a factor of two than the value $\mathrm{d} \delta / \mathrm{d} x \cong 0.012$ that holds for the far-field inner-layer growth rate of a wall jet on a smooth boundary (Launder \& Rodi 1983; George et al. 2000). George et al. showed that $\mathrm{d} \delta / \mathrm{d} x \propto u_{*}^{2} / U_{m}^{2}=c_{f} / 2$ in the far-field region of a wall jet, where $c_{f}$ is the skin-friction coefficient that is a function of Reynolds number $\operatorname{Re}_{\delta}=U_{m} \delta / \nu$. For a fully rough boundary layer of large Reynolds number, as in the present experiments, the skin friction coefficient is $c_{f} \cong 0.014$ (Grass 1971; HKGL), whereas in the far field region of a wall jet on a smooth wall $c_{f} \cong 0.007$ for $R e_{\delta} \approx 10^{4}$ (George et al. 2000). This difference in skin-friction coefficient is reflected in the difference in growth rate of the inner layer.

The attachment point of the jet at the free surface is at $x / b_{0} \approx 23$ (figure 2) and is equal to $x /\left(h_{2}-b_{0}\right) \approx 6.7$, a value close to that given for a backward-facing step at large Reynolds number. Downstream of this point, the velocity profile approaches the shape of an open-channel flow profile.

The dimensionless longitudinal mean velocity distributions and the mean vertical velocities are shown in figure 4 . Velocities are scaled with the maximum jet velocity $U_{m}$ and distance from the wall with $z_{1 / 2}$, that is the distance from the wall up to where $U=U_{m} / 2$. There is reasonably good collapse of the longitudinal velocity (figure $4 a$ ) in the lower part of the outer layer. In the upper part, the profiles do not collapse because of the variable return flow. Close to the wall there is considerable scatter in the measured velocities. With the acoustic profiler, measurements are accurate to a distance of $3 \mathrm{~mm}$ from the wall which is of the order of the wall roughness.

The vertical mean velocity, shown in figure $4(b)$, changes from negative values, when $x / b_{0}<8$, to positive values further downstream. Negative values indicate entrainment of ambient fluid into the jet. When the vertical velocity is positive, divergence of the flow dominates over entrainment. At $x / b_{0}=7.75$, the profile shows positive values 

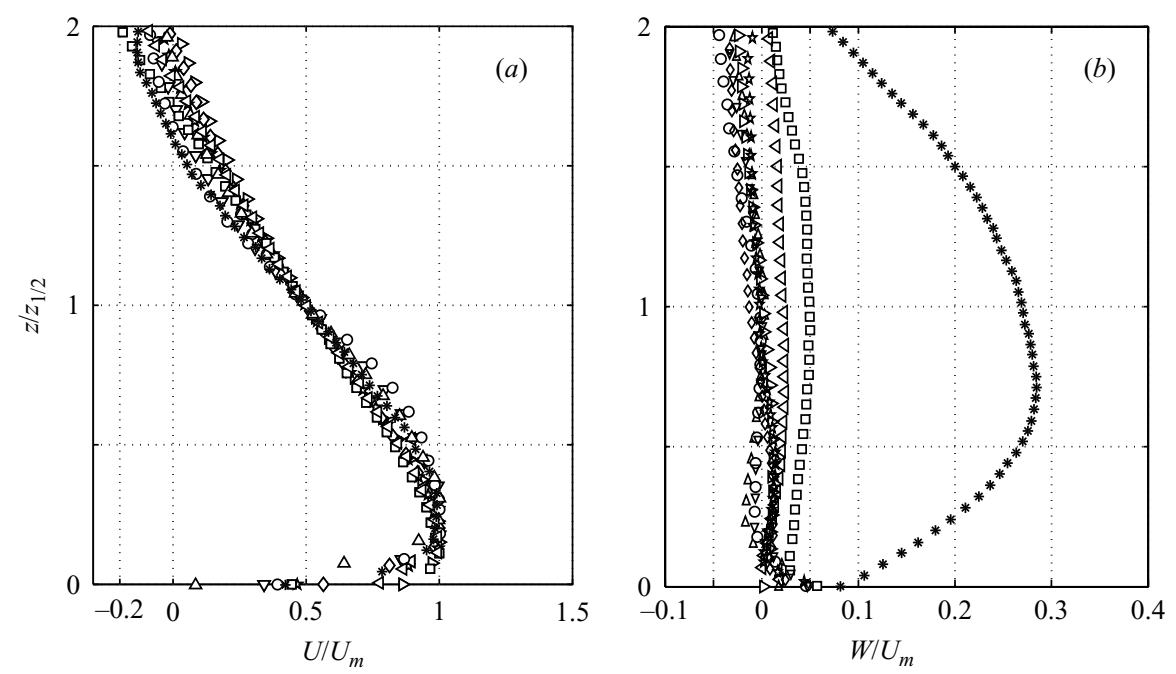

FIGURE 4. (a) Dimensionless mean longitudinal velocity profiles; (b) corresponding dimensionless vertical mean velocities. $\bigcirc, x / b_{0}=3.81 ; \nabla, x / b_{0}=4.75 ; \Delta, x / b_{0}=5.75$; $\triangleright, x / b_{0}=7.75 ; \diamond, x / b_{0}=8.75 ;$ 放 $x / b_{0}=9.75 ; \triangleleft, x / b_{0}=11.75 ; \square, x / b_{0}=13.75 ; *$, $x / b_{0}=18.75$.

for $z / z_{1 / 2}<1$ (divergence) and negative values above (entrainment). At $x / b_{0}=10$, the flow divergence dominates and increases with $x / b_{0}$. At $x / b_{0}=18.75$, the mean vertical velocity is very large. Therefore, this profile has not been included in the mean flow analysis.

\subsection{Scaling laws of the inner-layer mean flow evolution}

Hogg et al. (1997) introduced scaling laws based on the assumption that the initial streamwise momentum and the kinematic viscosity are the governing parameters. For the hydraulically rough wall that is of interest for sediment erosion, Hogg et al. determined the following power-laws for the evolution of maximum velocity and the boundary-layer thickness $\delta$ (position of velocity maximum):

$$
\begin{aligned}
U_{m} / U_{0} & =C_{3}\left(x / b_{0}\right)^{-m}\left(b_{0} / k_{e}\right)^{1-2 m}, \\
\delta / b_{0} & =C_{4}\left(x / b_{0}\right)^{n}\left(b_{0} / k_{e}\right)^{2(n-1)},
\end{aligned}
$$

where $C_{3}$ and $C_{4}$ are constants (the numbering of the coefficients is the same as in Hogg et al.), $k_{e}$ is the roughness length, proportional to grain size, here $k_{e}=d_{50}=2 \mathrm{~mm}$. Hogg et al. determined the constants and exponents from the data of Rajaratnam (1981). In the fit of (2) and (3) to the present data set shown in figure 5, we excluded the first two points that fall well within the potential core region. The best fit of the present data results in exponents $m=0.48$ and $n=0.82$, which is close to the values of $m=0.475 \pm 0.005$ and $n=0.84 \pm 0.025$ suggested by Hogg et al. (1997). The constants are $C_{3}=2.3$, which is equal to the value determined by Hogg et al., and $C_{4}=0.19$ which is close to their value of 0.2. However, if we use the Hogg et al. values for $m$ and $n(m=0.47, n=0.84)$, the constant $C_{4}$ reduces to $C_{4}=0.15$. The variation of $U_{m}$ and $\delta$ of the flow on the concave wall, also shown in figure 5, will be discussed in $\S 4$. The line fitted through the concave wall velocity data in figure $5(a)$ (dashed line) has the same exponent, but the constant is $C_{3}=1.53$. 

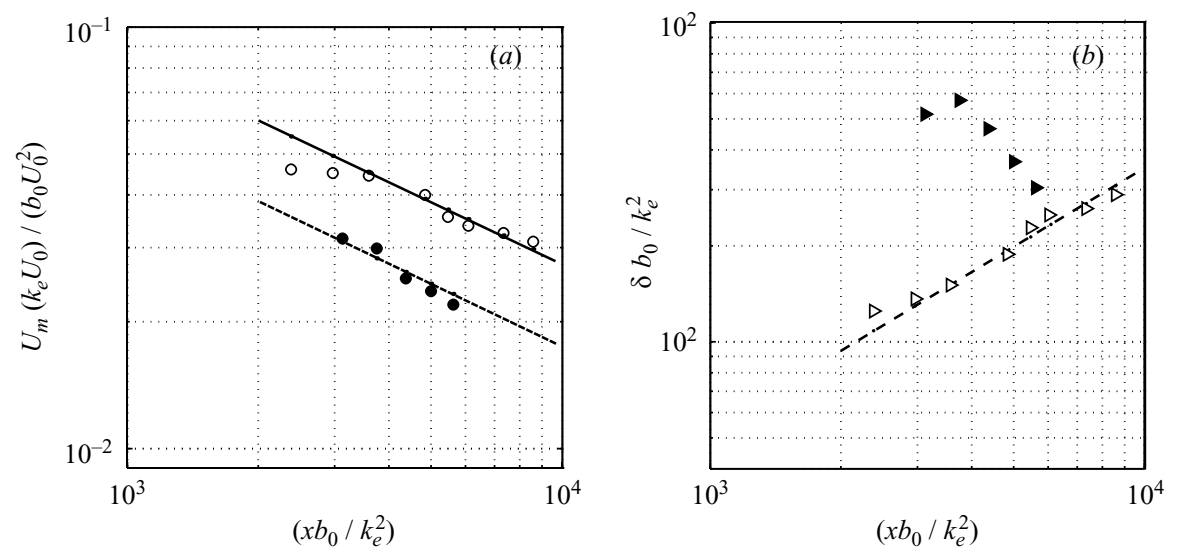

FIGURE 5. (a) Variation of scaled maximum velocity, $U_{m} k_{e} U_{0} /\left(b_{0} U_{0}^{2}\right)$ with $x b_{0} / k_{e}^{2}$ : , for the flat wall; $\bullet$, for the concave wall; and $(b)$ dimensionless boundary-layer thickness $\delta b_{0} / k_{e}^{2}$ as a function of $\delta b_{0} / k_{e}^{2} ; \triangleright$, for the flat wall; $\boldsymbol{\sim}$, for the concave wall. The two data points corresponding to $x / b_{0}<6$ were not taken into account in the fitting of the power law.

\subsection{Reynolds stresses}

In figure 6, the Reynolds stresses are scaled by the square of the maximum velocity $U_{m}^{2}$. The velocity $U_{s}=U_{m}-U_{r}$, where $U_{r}$ is the return flow caused by the confinement, could also be used as a characteristic velocity scale and this has been considered. However, $U_{s}$ is less well defined than $U_{m}$ and is not consistently representative of the mean shear, because the maximum of the return flow is not located at the same value of $z / z_{1 / 2}$. For this reason, $U_{m}$ was used as characteristic velocity.

It can be seen in figure 6 that the Reynolds stresses exhibit a rapid increase and change in distribution with downstream distance. Close to the nozzle, the maximum values would be expected to be bounded by the mixing-layer values and at large downstream distances, before flow reattachment at $x / b_{0} \approx 20$, by those of a plane free jet or by the Reynolds stresses in the flow over a backward-facing step. At intermediate downstream distances, the Reynolds stresses may be similar to those of a wall jet. For the physical interpretation of the flow development and for the sake of comparison, it is of interest to show in figure 6, together with the present data, the Reynolds stress distributions of a turbulent free shear layer and of a plane jet in addition to the far field of a wall jet. In the Appendix, it is shown that the measured Reynolds shear stress at $x / b_{0}=13.75$ is in good agreement with the flow over a backward-facing step.

Figure $6(a)$ indicates that the maximum Reynolds shear stress in the shear-layer region $x / b_{0}<6$ is located close to $z / z_{1 / 2}=1$, and the profile is close to that of a free shear layer. However, surprisingly at first sight, the maximum value is only half that of a free shear layer, indicated by the solid-line curve in figure 6(a) taken from Pope (2000). Further downstream, the shear stress progressively increases and the maximum value shifts to positions $z / z_{1 / 2}>1$, whereas in the far field of a turbulent wall jet on a smooth wall, the position of the maximum shear stress is at $z / z_{1 / 2}=0.75$ to 0.8 as indicated by the dashed-line curve (Karlsson, Ericsson \& Persson 1992; Zhou, Heine $\&$ Wygnanski 1996); the maximum values are comparable when $x / b_{0} \approx 8$. Only the position of the maximum is shifted outward. These maximum shear stress values are almost a factor of two smaller than the value in a free plane jet, as indicated by 

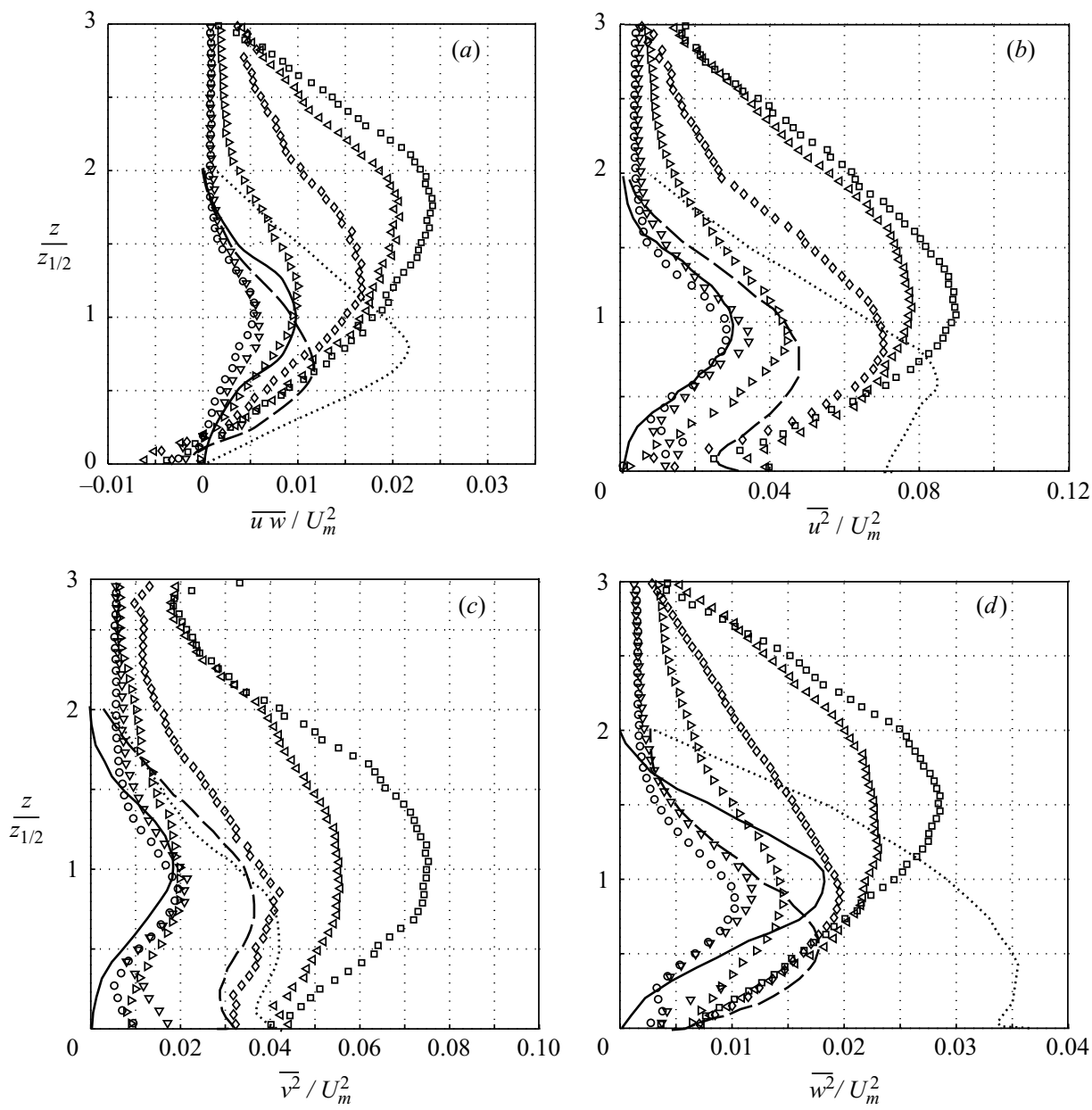

FIGURE 6. Turbulence quantities shown in terms of outer parameter scaling. (a) Reynolds shear stress $\overline{u w} / U_{m}^{2} ;(b)$ longitudinal normal Reynolds stress $\overline{u^{2}} / U_{m}^{2} ;(c)$ spanwise normal Reynolds stress $\overline{v^{2}} / U_{m}^{2} ;(d)$ cross-stream normal Reynolds stress $\overline{w^{2}} / U_{m}^{2}$. $\bigcirc, x / b_{0}=3.81 ; \nabla$, $x / b_{0}=4.75 ; \triangleright, x / b_{0}=7.75 ; \diamond, x / b_{0}=8.75 ; \triangleleft, x / b_{0}=11.75 ; \square, x / b_{0}=13.75 ;-$, free shear layer; - - , plane wall jet; ..., free plane jet.

the dotted line taken from Pope (2000). Launder \& Rodi (1983) mention that the reason for this lower value of the shear stress in the outer region of a wall jet as compared to the free plane jet, is probably due to the presence of the wall felt through pressure effects. The same reasoning can be used to explain the lower shear stress value in the mixing-layer region mentioned above. When $x / b_{0}>8$, the maximum shear stress further increases and reaches values comparable to a free plane jet. The presence of the strong return flow and the associated adverse pressure gradient that leads to positive mean vertical velocities (figure $4 b$ ) are the cause for the shift in the location and the increase in maximum shear stress compared to a wall jet; the wall effect on the outer-flow quantities seems to become insignificant when the mean flow is diverging. At $x / b_{0}=13.75$, the shear stress profile is in good agreement with that observed in the flow behind a backward-facing step (see Appendix). 


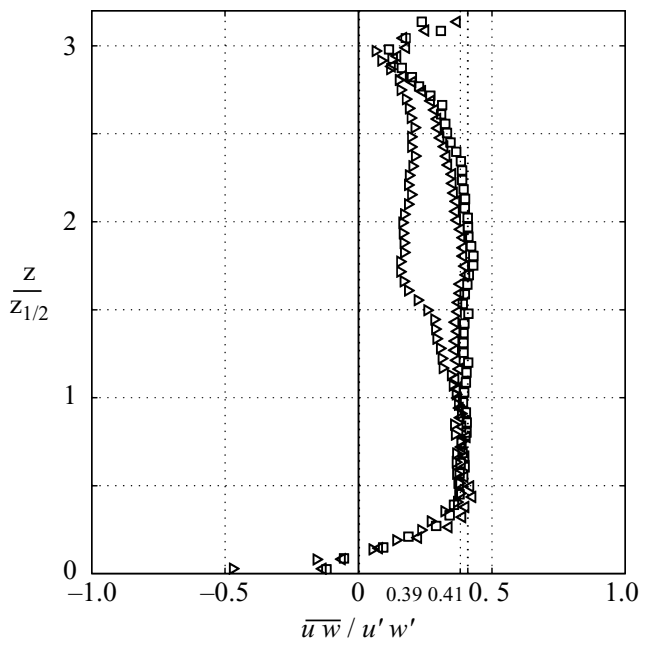

FIGURE 7. Reynolds stress correlation coefficient, $\overline{u w} / u^{\prime} w^{\prime}$, as a function of dimensionless distance from the wall. $\triangleright, x / b_{0}=7.75 ; \triangleleft, x / b_{0}=11.75 ; \square, x / b_{0}=13.75$.

The profiles of the normal Reynolds stresses, $\overline{u^{2}} / U_{m}^{2}, \overline{v^{2}} / U_{m}^{2}$ and $\overline{w^{2}} / U_{m}^{2}$, presented in figures $6(b),(c)$ and $(d)$, respectively, show a variation similar to the Reynolds shear stress in the outer layer. The maximum values are again located at positions $z / z_{1 / 2}$ larger than those reported for the far field of a turbulent wall jet on a smooth boundary, where the maxima are at $z / z_{1 / 2} \approx 0.7$ to 0.8 (Kobayashi \& Fujisawa 1983; Zhou et al. 1996; Erikson, Karlsson \& Persson 1998; George et al. 2000). In the inner layer, the longitudinal (streamwise) and spanwise normal Reynolds stresses, respectively $\overline{u^{2}} / U_{m}^{2}$ and $\overline{v^{2}} / U_{m}^{2}$, have a minimum around or below $z / z_{1 / 2}=0.4$. Previous studies (Kobayashi \& Fujisawa 1983; Erikson et al. 1998; George et al. 2000) also report clear minima of the longitudinal component in the inner layer. The cross-stream normal Reynolds stress (figure $6 d$ ) has a minimum closer to the wall at about $z / z_{1 / 2}=0.25$. The longitudinal normal Reynolds stress profile in the mixing-layer region, $x / b_{0}<6$, agrees well with the free shear-layer value, whereas the cross-stream normal Reynolds stress is only about half this value. The lower Reynolds shear stress is, therefore, due to the damping of the turbulence component normal to the wall. Relative to the two other components, the maximum of the cross-stream normal stress component in the free plane jet is large and located very close to the jet centre. This would indicate some flapping of the free jet that is prevented by the confinement of the wall jet.

Figure 7 shows the Reynolds stress correlation coefficient $\overline{u w} / u^{\prime} w^{\prime}$ as a function of dimensionless distance from the wall $\left(u^{\prime}\right.$ and $w^{\prime}$ are the r.m.s. values). In the outer layer, its value is close to 0.41 . This is $20 \%$ less than in the outer layer of a developed wall jet (Zhou et al. 1996). The variation in the wall layer and just above is practically the same as measured by Zhou et al. (1996). Zero Reynolds stress correlation is at $z / z_{1 / 2} \approx 0.12$, which is well below the position of the zero mean velocity gradient.

\subsection{Wall shear stress}

The integration of the streamwise momentum equation leads to the following expression for the calculation of the wall shear stress, $\tau_{b}$ (Hogg et al. 1997):

$$
\tau_{b}-\tau_{z=h}=-\frac{\mathrm{d}}{\mathrm{d} x} \int_{0}^{h} \rho u^{2} \mathrm{dy}-\Delta p .
$$




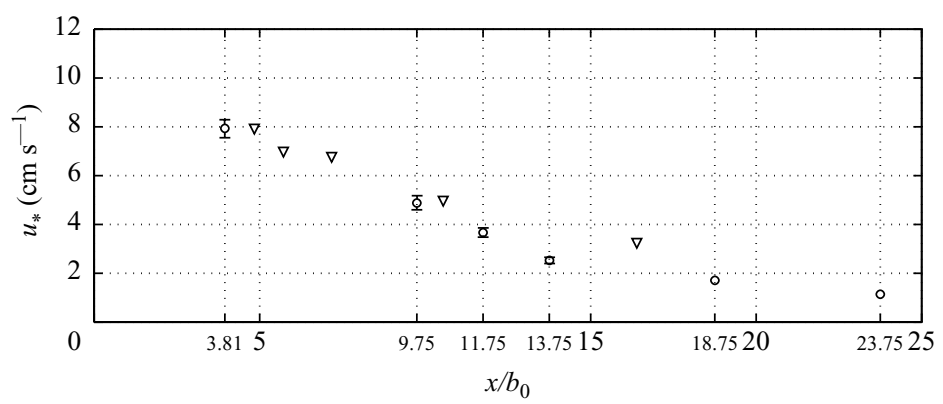

FIGURE 8. Dimensional friction velocity $u_{*}$ as a function of downstream distance. $\bigcirc$, values measured with hot-film; $\nabla$, from momentum balance.

The integration is from $z=0$ to $z=h$, where $h$ was chosen as the position where the mean velocity is equal to zero. The derivative of the streamwise component $(\partial / \partial x)$ is evaluated between two consecutive profiles in the flow direction. The pressuregradient effect is small on a flat wall and has been neglected in (4). As expected, the wall shear stress decreases with distance from the gate, mainly because $U_{m}$ decreases.

The wall shear stress was also measured with a hot-film probe at several downstream positions. The friction velocities, $u_{*}=\sqrt{\tau_{b} / \rho}$, measured with the hot-film probe and the one determined from the momentum balance are given in figure 8. Both follow the same trend. In accordance with $c_{f}=0.014$ for a fully rough wall (§3.1), the largest value of the dimensionless friction velocity is expected to be $u_{*} / U_{m} \cong 0.084$. Close to the gate the mean velocity is $U_{m}=1.14 U_{0}$ (figure 3), which gives $u_{*} \cong 6.74 \mathrm{~cm} \mathrm{~s}^{-1}$. This value is reasonably close to, but larger than, the measured value at $x / b_{0}=3.81$. The error in wall friction velocity measurements is evaluated to be $\pm 5 \%$. However, the dispersion in measured friction velocities indicates that the error is likely to be closer to $\pm 10 \%$. For comparison, it is useful to note that the critical Shields parameter for incipient motion of loose sand grains of $d_{50}=2 \mathrm{~mm}$ is $\tau_{c r i t}^{*}=\tau_{b} /\left(\rho_{s}-\rho\right) g d_{50}=0.035$, giving $\tau_{b}=1.13 \mathrm{~N} \mathrm{~m}^{-2}$ equal to $u_{*_{c}} \cong 3.4 \mathrm{~cm} \mathrm{~s}^{-1}$.

It is of interest here to represent the friction velocity in terms of the scaling proposed by Hogg et al. that includes dependency on roughness. The expression for $u_{*}$ is of the form

$$
u_{*} / U_{0}=\sqrt{C_{5}}\left(b_{0} / k_{e}\right)\left(x b_{0} / k_{e}^{2}\right)^{-m+n / 2-1 / 2} .
$$

where $C_{5}$ is a constant. In figure 9 , the ratio $u_{*} k_{e} / U_{0} b_{0}$ is plotted versus $x b_{0} / k_{e}^{2}$. The best fit gives $C_{5} \cong 0.13$. There is good agreement between the slope of the best-fit curve and the calculated values obtained from (5), taking $m=0.47$ and $n=0.84$ as previously determined ( $\$ 3.2)$. The last two points at $x / b_{0} \geqslant 18.75$ have not been taken into account in the least-squares fit, because the velocity profile shape changes rapidly.

The expression for $u_{*} / U_{m}$ is readily obtained from (2) and (5):

$$
u_{*} / U_{m}=\frac{\sqrt{C_{5}}}{C_{3}}\left(b_{0} / k_{e}\right)^{n-1}\left(x / b_{0}\right)^{n / 2-1 / 2} .
$$

This indicates a slow decrease of $u_{*} / U_{m}$ with downstream distance. Since the Reynolds number $\operatorname{Re}_{\delta}=U_{m} \delta / \nu$ increases as $\left(x / b_{0}\right)^{0.37}$, the slow decrease in $u_{*} / U_{m}$ with downstream distance is consistent with the variation in developed wall jets (George et al. 2000). Although we treat here the near-field flow structure that does not obey 


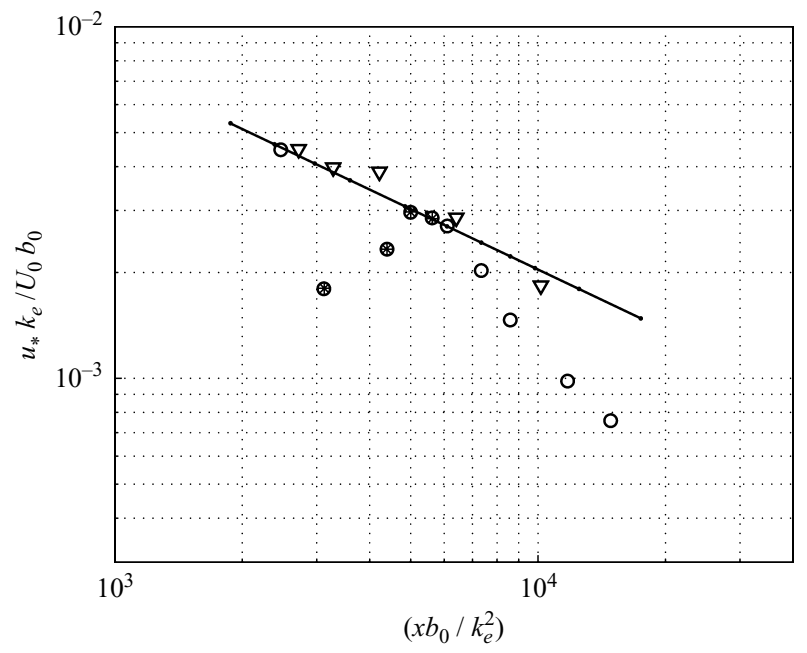

FIGURE 9. Variation of the scaled wall friction velocity versus dimensionless downstream distance. $\bigcirc$, values measured with hot-film; $\nabla$, momentum balance flat boundary; $\circledast$, values measured with hot-film on the concave wall. ----, equation (5) with $m=0.47, n=0.84$ and $C_{5}=0.13$.

similarity laws, many of the similarity concepts apply to the first order or at least give a basis for comparison.

\section{Flow structure on the concave wall}

\subsection{Mean flow quantities}

The velocity vectors measured along the concave wall of the shape shown in figure $1(b)$ are presented in figure 10. These profiles indicate a nearly separated flow on the downslope side (profile $1, x / b_{0}=3.81$ ), whereas at the upslope positions, especially profiles 3, 4 and 5, the mean shear is increased. When comparing the profiles with those on the horizontal boundary (figure 2), it can be seen that during the first rapid scouring phase of a mobile bed as defined by HKGL, the scour-hole length is within $x / b_{0} \leqslant 9$. This corresponds to a large portion of the potential core region on the flat wall. On the concave wall, the potential core is shorter and the velocity maximum has started to decrease by $x / b_{0} \approx 4$, mainly because of the increase in the flow crosssection and the associated adverse pressure gradient that causes the flow to nearly separate in the downslope region (see profile 1 , figure 10 ). At $x / b_{0}=5$, the velocity ratio is $U_{m} / U_{0} \cong 0.7$, compared with $U_{m} / U_{0} \cong 1.14$ at the same downstream location on a flat wall. For $x / b_{0}>5$, the decrease in maximum velocity is approximated well by (2) with the coefficient $C_{5} \approx 1.53$ instead of $C_{3}=2.3$ for the flat-wall flow (figure $5 a$ ). The position of the velocity maximum with respect to the wall decreases rather than increases with $x / b_{0}$. It varies from $\delta / b_{0} \approx 0.8$ at $x / b_{0}=5$ to $\delta / b_{0} \approx 0.48$ at $x / b_{0}=9$ (figure $5 b$ ). This is a consequence of the adverse pressure gradient related to the geometry.

The image of sediment transport on a mobile bed (figure 11), taken from HKGL, shows sediment ridges on the upslope side that are a signature of Görtler vortices. The Görtler number for the flow configuration as shown in figure 11, which corresponds closely to the present conditions, has been evaluated by HKGL and is about 10 times the critical value for the instability onset. 


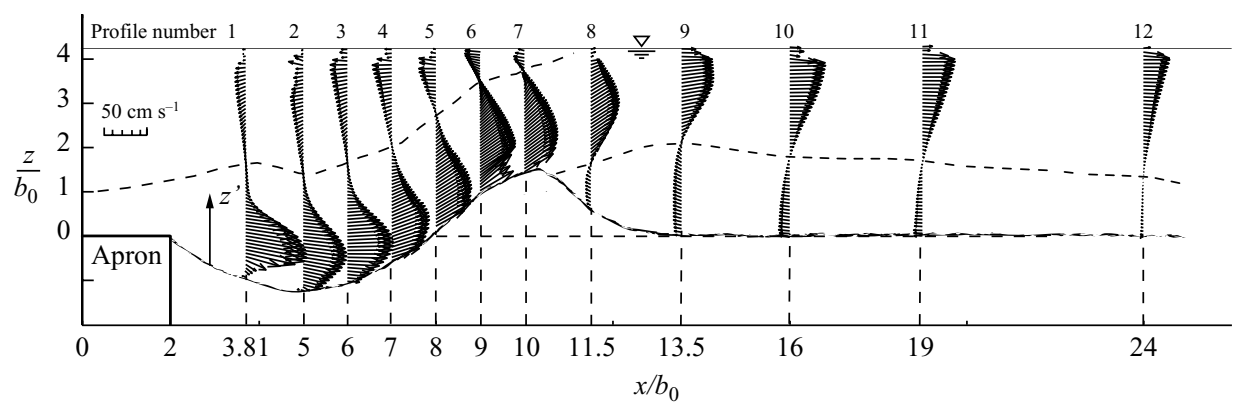

Figure 10. Mean velocity vectors in the $\left(x, z^{\prime}\right)$-plane on the concave wall. The position $x=0$ is at the sluice gate and the zero position of $z^{\prime}=z+h(x)$ is at the concave boundary, where $h$, a function of $x$, is measured from the position $z=0$ (figure $1 b$ ). The dashed line delineates backflow. The velocity scale on the left shows $\sqrt{U^{2}+W^{2}}$.

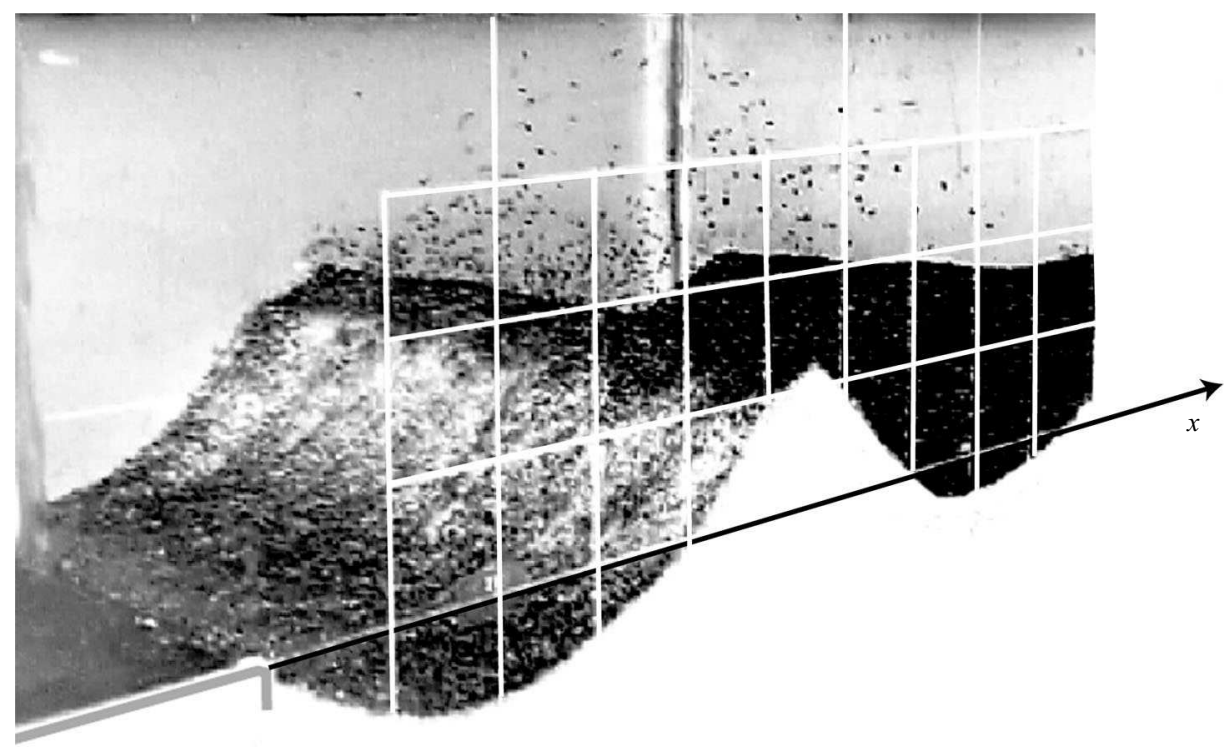

FIGURE 11. Image of sediment ridges (oblique, streamwise view of the sediment surface taken on a loose sediment boundary) caused by Görtler vortices (from HKGL). Note that the width of the channel is $50 \mathrm{~cm}$ and there are at least 7 longitudinal sediment ridges indicating longitudinal vortical structures of size of about $3 \mathrm{~cm}$. The grid spacing on the wall is $5 \mathrm{~cm}$.

\subsection{Instantaneous flow structure and turbulence quantities}

Figure 12 shows instantaneous turbulent velocity vectors in the $\left(x, z^{\prime}\right)$ - and the $\left(y, z^{\prime}\right)$ planes at $x / b_{0}=7$, measured in the centre of the channel in the time interval $\Delta t=t-t_{0}=1.6 \mathrm{~s}$. Within the measurement time of $T=180 \mathrm{~s}$, the time $t_{0}=40$ $\mathrm{s}$ was chosen such that upwash regions are emphasized. The Görtler vortices are unstable and move in a spanwise direction over distances of the order of their size. This spanwise movement allows us to identify the presence of coherent streamwise vortex structures by instantaneous velocity recordings in the centre of the channel. The vortices are about $3 \mathrm{~cm}$ and have typically an r.m.s. velocity of $10 \mathrm{~cm} \mathrm{~s}^{-1}$, giving an overturning time scale $t_{G} \approx 0.3 \mathrm{~s}$, hence $\Delta t / t_{G} \approx 5$. It is, therefore, possible to see in the time interval of $5 t_{G}$, a few, but not more than five, vortex signatures (figure 12). 

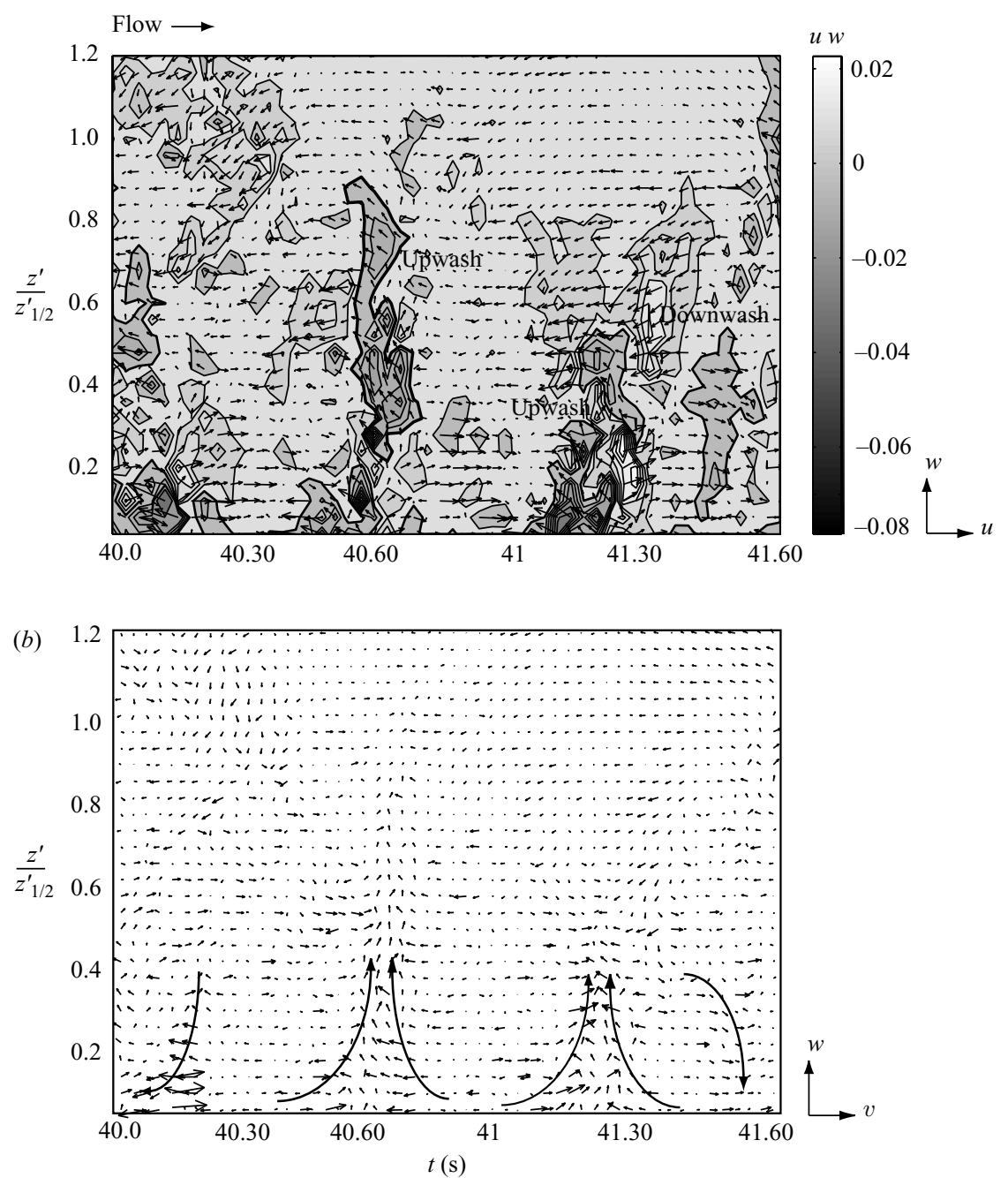

FIGURE 12. Two-dimensional turbulent velocity vector plots of profile $4\left(x / b_{0}=7\right)$ measured at the centre of the channel in the time interval $\Delta t=t-t_{0}=1.6 \mathrm{~s}$, where $t_{0}=40 \mathrm{~s} . U_{m}=0.527$ $\mathrm{m} \mathrm{s}^{-1}$ and $z_{1 / 2}^{\prime}=8.4 \mathrm{~cm}$ for profile 4 . (a) In the streamwise $\left(x, z^{\prime}\right)$-plane; $(b)$ in the spanwise $\left(y, z^{\prime}\right.$-plane $)$.

It should be emphasized that we see only the manifestation of streamwise vortices and not the vortex pattern because this would require the vortices to have a periodic spanwise movement over a distance of one wavelength (two vortex diameters); this is clearly not the case. In figure 12(a), such flow patterns as upwash and downwash regions in the $\left(x, z^{\prime}\right)$-plane are presented, together with the Reynolds shear stress distribution. Upwash events occur at $t=40.10,40.60$ and $41.30 \mathrm{~s}$ and can extend up to nearly $z^{\prime} / z_{1 / 2}^{\prime} \approx 1$ (at $t=40.60 \mathrm{~s}$ ). The contribution of upwash events to the Reynolds shear stress is significant. In the outer layer, the downwash and upwash flow events are dominant at several locations (for $t=40.30 \mathrm{~s}$ at $z^{\prime} / z_{1 / 2}^{\prime} \approx 1$ and for $t=41.60 \mathrm{~s}$ at $\left.z^{\prime} / z_{1 / 2}^{\prime} \approx 0.5\right)$. These observations suggest the existence of coherent vortices aligned with the mean flow and this is clearly demonstrated in figure $12(b)$ 


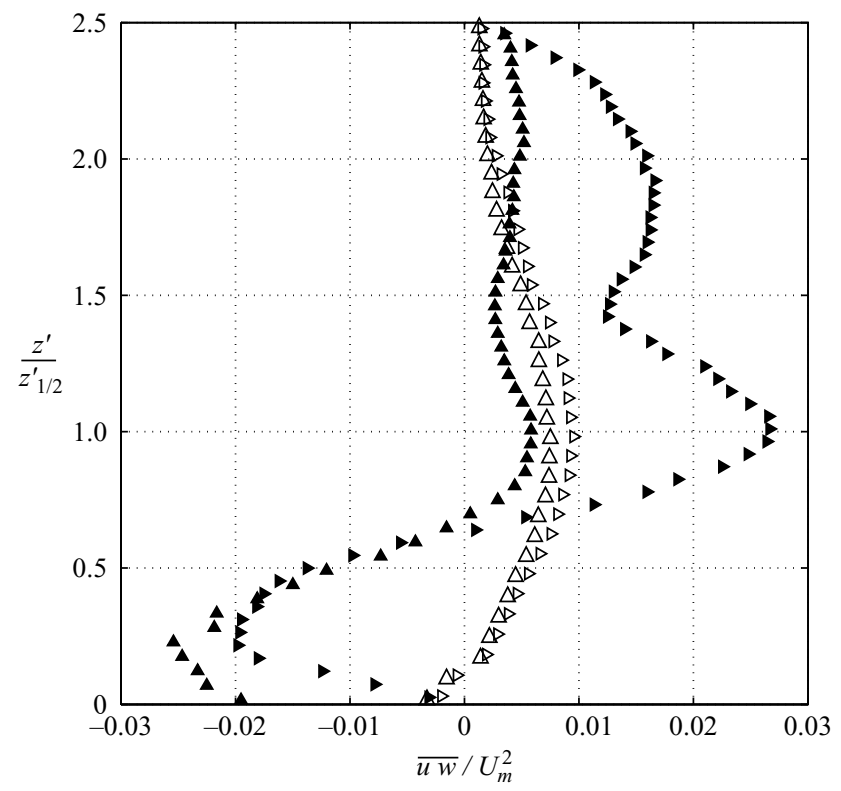

FIGURE 13. Dimensionless Reynolds shear stress profiles on the flat and concave wall at positions: $\Delta, x / b_{0}=4.75 ; \triangleright, x / b_{0}=7.75$, for the flat wall; and $\boldsymbol{\Delta}, x / b_{0}=6 ;-x / b_{0}=8$ for the concave wall. Note that $U_{m}$ is less on the concave wall than on the flat wall.

which shows the instantaneous velocity vectors at different times in the $\left(y, z^{\prime}\right)$-plane. The matching between $(u, w)$ and $(v, w)$ flow field patterns is quite remarkable.

In figure 13, the dimensionless Reynolds shear stress distributions on the concave wall are shown for two upslope flow profiles of downstream locations $x / b_{0} \approx 6$ and $x / b_{0} \approx 8$. The corresponding Reynolds stress distributions measured on the flat wall are included for comparison. It can be seen that the Reynolds shear stress values and profiles are drastically changed by the wall curvature. On the concave wall, the Reynolds shear stress is negative from the boundary up to $z^{\prime} / z_{1 / 2}^{\prime} \cong 0.7$ where it changes sign. This is above the position $\delta / z_{1 / 2}^{\prime} \approx 0.5$ where the mean velocity gradient changes sign from positive to negative and is opposite to what is observed for a wall jet on a flat wall, where zero Reynolds shear stress is below the position of maximum velocity (on the flat wall $h=0$, hence $z^{\prime} \equiv z$ ). This change in zero shear stress with respect to zero mean velocity gradient is indicative of the importance of the large-scale Görtler vortices on the concave wall. The minimum value of the shear stress (maximum absolute value) is located at $z^{\prime} / z_{1 / 2}^{\prime} \approx 0.3$ and is $\overline{u w} / U_{m}^{2} \cong 0.25$, which is about four times the shear stress on the flat wall. Unfortunately, very close to the wall (within about $3 \mathrm{~mm}$ above the roughness height) measurements with the acoustic profiler are not reliable. Nevertheless, the order of magnitude and the sign variation with $z^{\prime}$ are correct, as is indicated by the measurements on the flat wall shown in figure 6 . In the outer layer, above the velocity maximum, the shear stress is positive. Its value increases considerably with downstream distance from $x / b_{0}=6$ to $x / b_{0}=8$. The large value at $x / b_{0}=8$ is due to the change in the vertical mean flow component.

The profiles of the Reynolds stresses, $\overline{u^{2}} / U_{m}^{2}$ and $\overline{w^{2}} / U_{m}^{2}$, on the flat and concave wall are compared in figure 14. On the concave wall these longitudinal and crossstream normal Reynolds stresses are actually the $x$ and $z^{\prime}$ components and are not 

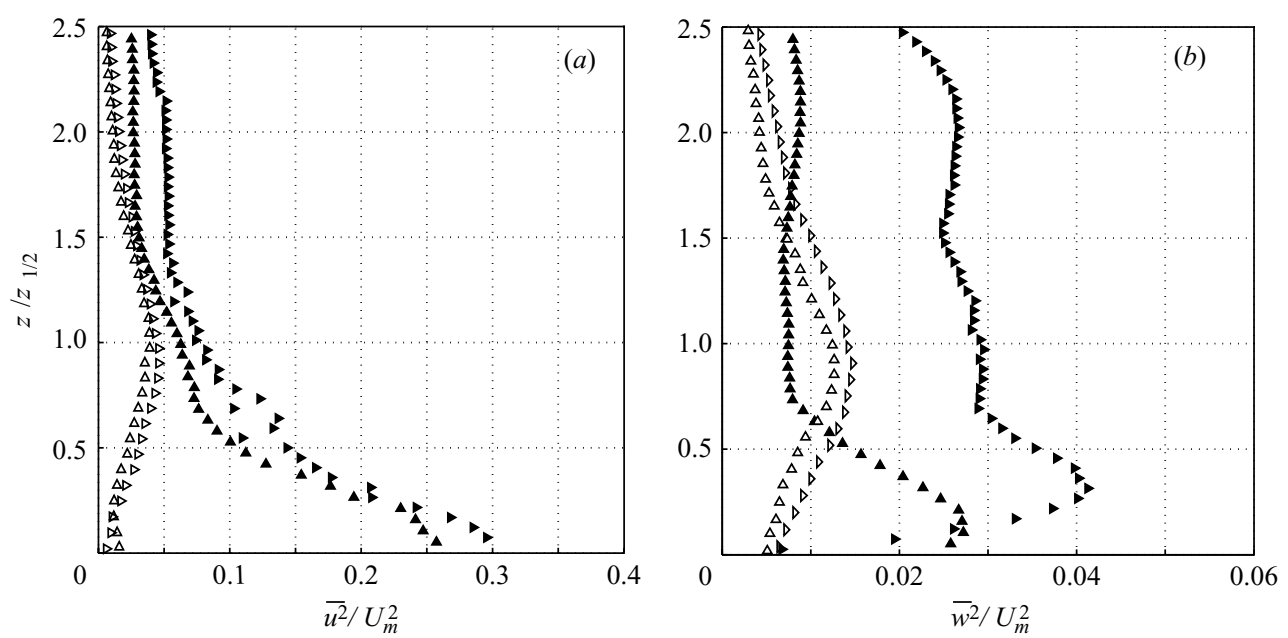

FIGURE 14. Dimensionless normal Reynolds stress profiles. (a) Longitudinal ( $x$-component) normal Reynolds stress $\overline{u^{2}} / U_{m}^{2}$, and $(b)$ cross-stream ( $z^{\prime}$ component) normal Reynolds stress $\overline{w^{2}} / U_{m}^{2}$. $\mathbf{\Delta}, x / b_{0}=6 ; \boldsymbol{\nabla}, x / b_{0}=8$, concave wall and $\Delta, x / b_{0}=4.75 ; \triangleright, x / b_{0}=7.75$ flat wall.

parallel and perpendicular to the wall. Nevertheless, we refer to these Reynolds stress components as longitudinal and cross-stream normal Reynolds stresses. Since the mean flow is not parallel to the wall, it would not make much sense to determine the stresses parallel and perpendicular to the wall. At $x / b_{0}=6$ for instance, the $x$ and $z^{\prime}$ components represent closely the longitudinal and cross-stream components.

In the inner layer, the longitudinal and cross-stream normal Reynolds stresses are considerably larger on the concave wall. On the flat wall, the maxima of longitudinal and cross-stream components are at $z^{\prime} / z_{1 / 2}^{\prime}=1$ (note that on the flat wall $h=0$, hence $z^{\prime} \equiv z$ ), whereas they are closer to the wall in the flow on the concave wall. The maximum of the longitudinal Reynolds stress is at $z^{\prime} / z_{1 / 2}^{\prime}<0.1$ and the cross-stream Reynolds stress maxima are at $z^{\prime} / z_{1 / 2}^{\prime}=0.2$ for profile $x / b_{0}=6$ and at $z^{\prime} / z_{1 / 2}^{\prime}=0.4$ for profile $x / b_{0}=8$. In the outer layer of the concave wall flow, the cross-stream Reynolds stress for profile $x / b_{0}=6$ is close to values on the flat wall, whereas for profile $x / b_{0}=8$, the value is 3 times larger. This large value is consistent with the shear stress and is attributed to the gradient of the mean vertical velocity resulting in production of cross-stream turbulence. The large values in Reynolds stresses in the inner layer are a signature of the Görtler vortices. These vortices reach up to $z^{\prime} / z_{1 / 2}^{\prime} \approx 0.7$ (figure $12 b$ ) and thus cause large velocity fluctuations.

The dimensionless wall friction velocities $u_{*} / U_{m}$, measured with the hot-film probe on the concave wall are plotted in figure 15 . The values obtained with the hot-film probe and from momentum balance on the flat wall are included for comparison. It can be seen that at $x / b_{0}=5$, the dimensionless friction velocity $u_{*} / U_{m}$ on the concave wall is below the value of the flat wall, but it exceeds the value on the flat wall when $x / b_{0}>7$. This higher dimensionless friction velocity on the concave wall, when $x / b_{0}>7$, can be attributed to the development of Görtler vortices. When $x / b_{0}>9$, the friction velocity on the concave wall starts to decrease, because after that point the boundary slope changes (figure 10) and the Görtler vortices disappear.

The observed wall shear stress on the concave wall does not follow the Hogg et al. model where it was assumed that the wall shear stress on a mobile bed decreases as the scour hole deepens. The expression for the bed or wall shear stress in the 


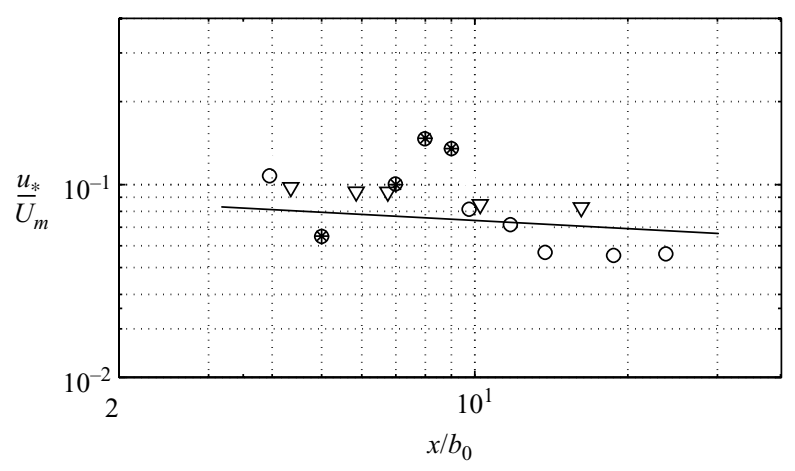

FIGURE 15. Dimensionless friction velocity, $u_{*} / U_{m}$, as a function of downstream distance, $\bigcirc$ and $\nabla$, values measured with hot-film probe and obtained from momentum balance on the flat wall; $\circledast$, values measured with hot-film on the concave wall. —-, equation (6) with $m=$ $0.47, n=0.84, C_{3}=1.53$ and $C_{5}=0.13$.

Hogg et al. model is

$$
\tau_{b}=C_{5} \rho U_{0}^{2}\left(b_{0} / k_{e}\right)^{2}\left(x b_{0} / k_{e}^{2}\right)^{-2 m+n-1} G\left(h, x^{\prime}\right),
$$

where the function $G(h, x)=1$ when $h=0$ and $G(h, x)=\exp \left(-(h / C \delta)^{2}\right)$ when $h>$ $0 ; \delta(x)$ is the boundary-layer thickness, (3), and $C$ is a constant. This Gaussian shape function allowed the scouring to come to a stop (forcing the wall shear stress to fall below the critical Shields value) when the scour hole reached the quasi-steady shape shown in figure $1(b)$. The present experiments indicate that this is true only for the downslope-flow region in the scour hole where the flow is nearly separating. At downstream locations beyond the distance of maximum scour hole depth, at $x / b_{0}>7$, the wall shear stress $\tau_{b} / \rho U_{0}^{2}$ remains nearly identical to the value on the flat wall (figure 9) and the dimensionless value $u_{*} / U_{m}$ actually increases (figure 15).

Since, on the concave wall, the dimensionless friction velocity $u_{*} / U_{m}$ reaches a value of about 1.5 times the value on a flat wall, the wall shear stress due to (turbulent) Görtler vortices increases by a factor of about two. Previous measurements (Barlow \& Johnston 1984) suggest a $40 \%$ increase in wall shear stress owing to Görtler vortices on a concave smooth wall (Floryan 1991). The increase will, of course, depend on the strength of the Görtler vortices and, to some extent, on whether the wall is rough or smooth. The interest of the present measurements is to show that the increase in Reynolds shear stress just above the bed (where our measurements are reliable) is considerably larger than the increase in wall shear stress (figure 13), thus, significantly increasing the momentum and scalar or sediment transport capacity.

\subsection{Evaluation of the contribution of the Görtler vortices to the shear}

HKGL assumed that the shear stress contributions by the shear-generated turbulence and the turbulence due to the Görtler vortices are additive (as turbulent energies are additive). The wall shear stress can thus be written in the form

$$
\tau_{b}=\frac{1}{2} \rho U_{m}^{2}\left(c_{f}+c_{f G}\right),
$$

where $c_{f}=0.014$ is the friction coefficient on a fully rough wall and $c_{f G}$ is the friction coefficient of the turbulence of the Görtler vortices. HKGL evaluated the turbulent Görtler vortex velocity to be $u_{G} \approx 0.25 U_{m}$ (this is consistent with the measurements shown in figure 14b) and assumed the Görtler shear stress to be $\tau_{G} / \rho \approx 0.3 k$ where 
$k \approx u_{G}^{2}$ is the Görtler vortex kinetic energy. This gives

$$
\tau_{G} / \rho \approx 0.019 U_{m}^{2} \text {. }
$$

The maximum Görtler vortex shear stress is thus about 2.7 times the turbulent bed shear stress, giving a total maximum shear stress of

$$
\tau / \rho \approx 0.007(1+2.7) U_{m}^{2}
$$

This is consistent with the measurements (figure 13) and is slightly less than the evaluation in HKGL where it was assumed that $k=3 u_{G}^{2} / 2$.

\section{Conclusions and further discussion}

The mean velocity and Reynolds stress measurements made with the ADVP allowed us to characterize the complex flow structure of a plane jet issuing from a sluice gate onto a flat and a concave wall consisting of fixed sand beds of surface roughness $k_{e}=d_{50}=2 \mathrm{~mm}$. The flow is strongly confined $\left(h_{2} / b_{0}=4\right)$ so that in the flat-wall configuration, the jet flow reattaches at the free surface before it reaches self-similarity. On the concave wall, self-similarity is not expected.

On the flat wall, the spatial growth rate of the outer layer and the maxima of the Reynolds stresses approach values accepted for the far field of a wall jet at a downstream distance $x / b_{0} \approx 8$. These maxima are only about half the values of a free plane jet and the same reduction is observed in the mixing-layer region, $x / b_{0}<6$, where the Reynolds shear stress is only about half the value of a free shear layer. At larger downstream distances, $x / b_{0}>11$, the maximum Reynolds shear stress approaches maximum values that are comparable with a plane free jet or, more precisely, comparable with the Reynolds stress distribution in the flow behind a backward-facing step (see Appendix). This change in the maxima of the Reynolds stresses is related to the mean vertical velocity that is negative for $x / b_{0}<8$ and positive beyond. This indicates that when $x / b_{0}<8$, the presence of the wall has a strong effect even on the outer-layer flow by reducing the normal turbulent fluctuations through pressure effects. The evolution of the inner region of the wall jet is found to be in good agreement with the Hogg et al. (1997) model that explicitly includes the roughness length. The wall shear stress measurements, essential for the evaluation of erosion capacity, are consistent with this model that predicts a slow decrease in the dimensionless friction velocity $u_{*} / U_{m}$ with downstream distance. This happens because the skin friction coefficient decreases with increasing Reynolds number $\operatorname{Re}_{\delta}=U_{m} \delta / v$ (George et al. 2000). As shown in the Appendix, the Reynolds stress distribution at the downstream location of four step heights is in good agreement with numerical simulations by Diurno, Balaras \& Piomelli (2001). Here, measurements are made much closer to the step and this could be of interest to modellers.

On the concave wall, the mean flow and the Reynolds stresses are drastically changed by the adverse pressure gradient and the development of Görtler vortices. The shape of the concave wall corresponds to the quasi-steady scour-hole shape of a mobile bed (HKGL). The maximum mean flow velocity decreases rapidly close to the gate because of the adverse pressure gradient; then, it decreases in a way similar to that on the flat wall. On the downslope side of the scour hole, the flow is nearly separating and the wall shear stress tends to zero. On the upslope side, the dimensionless wall shear stress, proportional to $\left(u_{*} / U_{m}\right)^{2}$, increases by a factor of about two with respect to the flat-wall value, owing to the development of Görtler vortices. These vortices extend well into the outer layer away from the wall. Just above the wall, the absolute 
value of the Reynolds shear stress substantially increases in accordance with the conjecture of $\mathrm{HKGL}$ and changes sign at $z^{\prime} / z_{1 / 2}^{\prime} \cong 0.7$ which is above the position of the maximum of the mean velocity; that behaviour is opposite to that observed for the wall jet on a flat wall. This is an indication of the dominant role of large-scale Görtler vortices in the wall layer. The increase in Reynolds shear stress in the scour hole increases the sediment transport capacity on a mobile bed. This is opposite to the Hogg et al. model, where it was assumed that the wall shear stress decreases in the form of a Gaussian-like shape function as the scour hole deepens and falls below the erosion value when the scour-hole shape has reached the quasi-steady state. The present results indicate that the dimensional wall shear stress remains practically constant and that the dimensionless friction velocity $u_{*} / U_{m}$ actually increases on the concave wall. Nevertheless, on a mobile bed, the effective transport goes almost to zero owing to a near equilibrium between upslope sediment transport and downslope avalanching when the slope angle exceeds the angle of repose. This can be modelled by a decreasing effective wall shear stress as the scour-hole depth increases.

The question arises as to whether or not the flow structure, measured on the concave fixed bed, is representative of the flow field on a mobile bed. As mentioned in $\S 2.2$, measurements with the ADVP system on a mobile bed are not reliable in the near-wall region because of the strong backscatter from the large moving and suspended particles. Most other techniques also fail in sediment-laden flows. The turbulence characteristics measured on the fixed bed can be considered as upper bounds on the Reynolds stresses including the Görtler vortices. On a mobile bed, the Görtler vortices are still present, as is demonstrated by the sediment ridges shown in figure 11. However, kinetic energy is expended when sediment is moved and lifted up, and the turbulence intensity near the wall may be reduced. Thus, the turbulence intensity and Görtler vortex strength are expected to be more intermittent on a mobile bed with peak values, as measured on the fixed bed, alternating with lower values in between.

The authors acknowledge the financial support of the Swiss National Science Foundation (grant 200020-100383). E. J. H. acknowledges a visiting scientist support grant by ERCOFTAC.

\section{Appendix. Backward-facing-step analysis of the flow on a horizontal wall}

The near-field flow structure can be analysed in terms of the scaling of a backward-facing step. The experimental configuration is shown in figure 16. Here, the characteristic scale is the step height $H_{e}=h_{2}-b_{e}$, where $b_{e}$ is the effective inflow jet thickness. Flow rate measurements downstream of the reattachment point at $x / b_{0}>24$, indicate that the inlet flow rate is $Q=300 \mathrm{~cm}^{2} \mathrm{~s}^{-1}$ per unit width. Therefore, the flow contraction of the jet emerging from the sluice gate is $b_{e} / b_{0}=Q / U_{0} b_{0} \approx 0.85$. The measured effective jet velocity is $U_{e}=1.14 U_{0}$.

Figure 16 shows the evolution of the non-dimensional mean velocity profiles at various downstream locations. Note that with respect to figure 2, the flow has been turned upside down, so that the flow of thickness $b_{e}$ issues above the step of height $H_{e}$. The lower boundary (that is the free surface) has nearly free-slip conditions. The zero velocity locations of the different profiles are shifted by $U / U_{e}=0.5$, with the zero of the last profile being at $U / U_{e}=3$. This representation is the same as in Diurno et al. (2001). In their study, the reattachment of the flow was emphasized, whereas here, all the profiles shown are in the backflow region. 


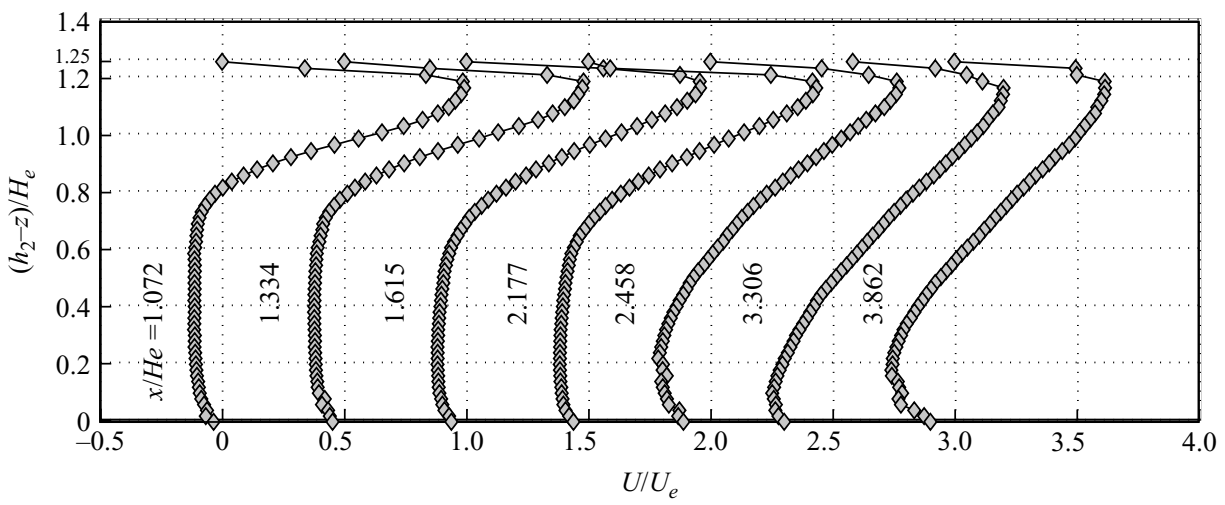

FIGURE 16. Profiles of mean velocities normalized by $U_{e}$. The zero velocity origin of the different profiles is shifted by $U / U_{e}=0.5$.

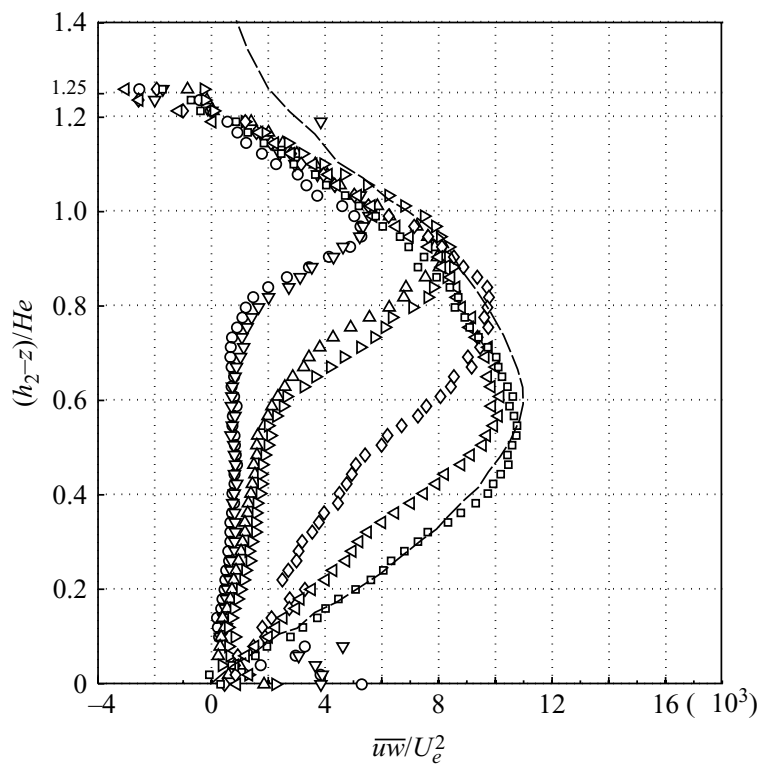

FigURE 17. Reynolds shear stress, $\overline{u w} / U_{e}^{2}$ in outer scaling. $\bigcirc, x / b_{0}=3.81 ; \nabla, x / b_{0}=4.75 ; \Delta$, $x / b_{0}=5.75 ; \triangleright, x / b_{0}=7.75 ; \diamond, x / b_{0}=8.75 ; \triangleleft, x / b_{0}=11.75, \square, x / b_{0}=13.75 ;-\cdots$, data from Diurno et al. (2001).

Figure 17 shows the Reynolds shear stress normalized by $U_{e}^{2}$. The last profile at $x / H_{e}=3.85$, corresponding to $x / b_{0}=13.75$, is close to the profile at $x / h=4.2$, with $h \equiv H_{e}$ of Diurno et al. (2001). However, in the present case, there is an upper cutoff because of the small dimensions of the flow (jet) above the step. The maximum non-dimensional Reynolds stress at $x / H_{e}=3.85$ of $\overline{u w} / U_{e}^{2} \cong 0.0011$ is practically identical to the value of Diurno et al., and Eaton \& Johnston (1980), in spite of the decreasing outer-flow velocity $U_{m}$. The reason for this is that the mean gradient is the same as in Diurno et al.

\section{REFERENCES}

Abrahamsson, H., Johansson B. \& LöFdahl, L. 1994 A turbulent plane two dimensional wall jet in a quiescent surrounding. Eur. J. Mech. Fluids. 13, 533-556. 
AlbayraK, I. 2007 Secondary currents and coherent structures, their distribution across a cross section and their relation to surface boils in turbulent gravel-bed open-channel flow. 32nd IAHR Congress, Venice, Italy, vol. 2, p. 767.

AlbayraK, I. \& Lemmin, U. 2007 Dynamics of secondary currents and surface boils in turbulent open-channel flow revealed by surface particle image velocity measurements. 32nd IAHR Congress, Venice, Italy, vol. 2, p. 486.

Barlow, R. S. \& Johnston, J. P. 1985 Roll-cell structure in a concave turbulent boundary layer. AIAA Paper 85-0297.

Chatterjee, S. S., Ghosh, S. N. \& Chatterjee, M. 1994 Local scour due to a submerged horizontal jet. J. Hydraul. Engng. ASCE 120, 973-992.

Diurno, G. V., Balaras, E. \& Piomelli, U. 2001 Wall-layer models for LES of separated flows. ERCOFTAC Bull. 48, 37-40.

Eaton, J. K. \& Johnston, J. P. 1980 Turbulent flow re-attachment: an experimental study of the flow and structure behind a backward-facing step. Stanford University, Rep. MD-39.

Eriksson, J., Karlsson, R. I. \& Persson, J. 1998 An experimental study of a two dimensional plane wall jet. Exp. Fluids 25, 50-60.

Floryan, J. M. 1991 On Görtler instability of boundary layers. Prog. Aerospace Sci. 28, 235-271.

Franca, M. \& Lemmin, U. 2005 Eliminating velocity aliasing in acoustic Doppler velocity profiler data. Meas. Sci. Technol. 16, 1-10.

George, W. K., Abrahamsson, H., Eriksson, J., Löfdahl, L., Karlsson, R. I. \& Wosnik, M. 2000 A similarity theory for the turbulent plane wall jet without external stream. J. Fluid Mech. 425, 367-411.

Grass, A. J. 1971 Structural features of turbulent flow over smooth and rough boundaries. J. Fluid Mech. 50, 233-255.

Hogg, A. J., Huppert, H. E. \& Dade W. B. 1997 Erosion by planar turbulent wall jets. J. Fluid Mech. 338, 317-340.

Hopfinger, E. J., Kurniawan, A., Graf, W. H. \& Lemmin, U. 2004 Sediment erosion by Görtler vortices: the scour-hole problem. J. Fluid Mech. 520, 327-342.

Hurther, D. \& Lemmin, U. 2000 A correction method for turbulence measurements with a 3D acoustic Doppler velocity profiler. J. Atmosph. Ocean. Technol. 18, 446-458.

Karlsson, R. I., Ericsson, J. \& Persson, J. 1992 LDV measurements of a plane jet in a large enclosure. Internal Rep. Department of Hydromechanics, Royal Institute of Technology, Sweden.

Kobayashi, R. \& Fujisawa, N. 1983 Turbulence measurements in wall jets along strongly concave surfaces. Acta Mech. 477, 39-52.

Kravchenko, A. G., Choi, H. \& Moin, P. 1993 On the relations of near-wall streamwise vortices to wall skin friction in turbulent boundary layers. Phys. Fluids A 5, 3307-3309.

Launder, B. E. \& Rodi, W. 1981 The turbulent wall jet. Prog. Aerospace Sci. 19, 81-128.

LAUnder, B. E. \& Rodi, W. 1983 The turbulent wall jet-measurements and modelling. Annu. Rev. Fluid Mech. 15, 429-459.

Lhermitte, R. \& Lemmin, U. 1994 Open-channel flow and turbulent measurement by high-resolution Doppler sonar. J. Atmos. Ocean. Technd. 11, 1295-1308.

Mason, P. J. \& ARumugam, K. 1985 Free jet scour below dams and flip buckets. J. Hydraul. Engng. ASCE 111, 220-235.

Mayle, R. E., Blair, M. F. \& Kopper, F. C. 1979 Turbulent boundary layer heat transfer on curved surfaces. Trans. ASME J. Heat Transfer 92, 521-525.

Nielsen, P. 1992 Coastal Bottom Boundary Layers and Sediment Transport. World Scientific.

Nino, Y. \& GARCIA, M. H. 1996 Experiments on particle-turbulence interactions in the near-wall region of an open-channel flow: implications for sediment transport. J. Fluid Mech. 326, 285-319.

Pope, S. B. 2000 Turbulent Flows. Cambridge University Press.

Rajaratnam, N. 1967 Hydraulic jumps. In Advances in Hydroscience (ed. V. T. Chow), pp. 197-280. Academic.

Rajaratnam, N. 1981, Erosion by plane turbulent jets. J. Hydraul. Res. 19, 339-358.

Rolland, T. \& Lemmin, U. 1997 A two-component acoustic velocity profiler for use in turbulent open-channel flow. J. Hydraul. Res. 35, 545-561. 
Shen, C., Song T. \& Lemmin, U. 1998 Skin friction measurement in variable temperature flow. Proc. IEEE Instrumentation and Measurement Technology Conf. Brussels. pp. 523-526.

Sumer, B. M., Cokgor, S. \& Fredsoe, J. 2001 Suction removal of sediment from between armor blocks. J. Hydr. Eng. 4, 293-206.

Tamburrino, A. \& Gulliver, J. S. 1999 Large flow structures in a turbulent open channel. $J$. Hydraul. Res. 37, 363-380.

Wygnanski, I., Katz Y. \& HoREv, E. 1992 On the applicability of various scaling laws to the turbulent wall jet. J. Fluid Mech. 234, 669-690.

Zhou, M. D., Heine C. \& Wygnanski, I. 1996 The effects of excitation on the coherent and random motion in a plane wall jet. J. Fluid Mech. 310, 1-37. 